\title{
Forecasting US Output Growth with Large Information Sets
}

\author{
Afees A. Salisu ${ }^{1}$, Umar Bida Ndako ${ }^{2}$ and Rangan Gupta ${ }^{3, *}$ \\ ${ }^{I}$ Centre for Econometric \& Allied Research, University of Ibadan, Ibadan, Nigeria. \\ ${ }^{2}$ Economic Policy Directorate, Central Bank of Nigeria. \\ ${ }^{3}$ Economics Department, University of Pretoria, South Africa.
}

\begin{abstract}
We forecast US output growth using an array of both Classical and Bayesian models including the recently developed Dynamic Variable Selection prior with Variational Bayes [DVSVB] of Koop and Korobilis (2020). We accommodate over 300 predictors that are incrementally captured from 5 factors, 60 factors to over 300 factors covering relevant economic agents. For robustness, we allow for both constant and time varying coefficients as well as alternative proxies for output growth. Using data covering 1960:Q1 to 2018:Q4, our results consistently support the use of high-dimensional models when forecasting US output growth regardless of the choice of forecast measure. For the density forecast of real GDP growth in particular, the results favour the DVSVB and time varying parameter assumption.
\end{abstract}

Keywords: US Output Growth; High-Dimensional Models; Forecast Evaluation.

JEL Codes: O41, O51, C52, C51, C53.

\section{INTRODUCTION}

Information about growth forecast help policymakers and investors make better decisions. From policy perspective, the expenditure framework of a typical economy whether in the short or medium term relies on the projections made for output growth. If an economy is projected to plunge into recession, policy makers may consider implementing expansionary fiscal and monetary policies (higher spending financed by borrowing and not higher taxes and lower interest rate, among others) to stimulate economic activities. From investment perspective, growth forecast serves as a barometer for gauging the potential of an economy to absorb and retain capital and investors are usually in search for economies with sustainable growth potential given its link with other macroeconomic fundamentals such as interest rate inflation and unemployment, among others.

Several attempts have been made in the literature to evaluate growth forecast (see Aromi, 2019 for a review) with alternative predictors suggested to improve the forecasts (see Baghestani \& AbuAl-Fou, 2017; Ercolani, 2020, for a review). We however hypothesize in this study that models that incorporate some details about the relevant economic agents namely households, businesses and the governments in the estimation process will produce better growth forecasts than other alternative models with less details. This is the main contribution of the study and we differ from the literature in terms of the variants of variables, models and estimators used in forecasting US real GDP growth. We consider an array of models where the Autoregressive model

*Address correspondence to this author at Economics Department, University of Pretoria, South Africa. E-mail: rangan.gupta@up.ac.za; serves as the benchmark model and thereafter we introduce predictors incrementally starting from a small number of predictors (5 factors) to a large set of predictors (over 300 of them).1 For completeness, we assume constant and time varying coefficients and based on model peculiarities, we also employ alternative estimators involving both Classical and Bayesian methods such as OLS, Partial Least Squares regression, MCMC algorithm (George and McCulloch, 1993), Bagging algorithm (Breiman (1996), Dynamic Model Averaging algorithm (Koop and Korobilis, 2012); and Dynamic Variable Selection prior with Variational Bayes (DVSVB) (Koop and Korobilis, 2020). Overall, our results support the use of high-dimensional models when forecasting US real GDP growth under certain conditions.

The next section provides the methodology followed by the presentation and discussion of results in Section 3 while Section 4 concludes the paper.

\section{METHODOLOGY AND DATA}

As noted earlier, we consider thirteen models as follows: (i) AR: Autoregressive model with constant parameter assumption but without predictors, estimated with OLS; (ii) TVPAR: an extension of AR model but with time varying parameter assumption and stochastic volatility estimated with MCMC; (iii) FAC5: an extension of AR model with five factors estimated with OLS; (iv) BAG/FAC5: same predictors as FAC5 but estimated as constant parameter regression using Bagging algorithm of Breiman (1996); (v) DMA/FAC5: same predictors as FAC5 but estimated as TVP

\footnotetext{
${ }^{1}$ See Koop and Korobilis (2020) for a detailed description of the predictors.
} 
regression using Dynamic Model Averaging algorithm of Koop and Korobilis (2012); (vi) DVSVB/FAC5: same predictors as FAC5, estimated as TVP regression using Dynamic Variable Selection prior with Variational Bayes (DVSVB) proposed by Koop and Korobilis (2020); (vii) GPR/FAC5: same predictors as FAC5, estimated as a Gaussian Process Regression; (viii) SSVS/FAC60: an extension of the benchmark AR specification with first 60 principal components, estimated using an SSVS prior with MCMC of George and McCulloch (1993); (ix) ELN/FAC60: same predictors as SSVS/FAC60, estimated as a constant parameter regression using the Elastic Net algorithm of Zou and Hastie (2005); (x) DVSVB/FAC60: same predictors as SSVS/FAC60, estimated as a TVP regression using Koop and Korobilis (2020) approach; (xi) ELN/X: builds on benchmark AR specification by augmenting it with 303 predictors, estimated using the Elastic Net algorithm of Zou and Hastie (2005); (xii) $\mathrm{PLS} / \mathrm{X}$ has the same predictors as in ELN/X, estimated as a constant parameter Partial Least Squares regression; (xiii) VBDVS/X has the same predictors as in ELN/X, estimated as a TVP regression using Koop and Korobilis (2020) approach. ${ }^{2}$ The generic representation of the predictive model is specified as follows:

$g_{t+h}=\alpha_{t}+\phi_{1, t} g_{t}+\phi_{2, t} g_{t-1}+x_{t} \beta_{t}+\varepsilon_{t+h}$

where $g_{t+h}$ denotes $h$-step ahead forecast for the real GDP growth; $x_{t}$ is a vector of growth predictors; $\alpha_{t}, \phi_{1, t}, \phi_{2, t}$ and $\beta_{t}$ are the model parameters corresponding respectively to the time varying intercept, coefficients of the two own lags and exogenous predictors; while $\varepsilon_{t+h}$ is the $h$-step ahead disturbance term. For constant parameter assumption,

$\alpha_{t}=\alpha ; \phi_{1, t}=\phi_{1} \ldots \phi_{2, t}=\phi_{2}$ and $\beta_{t}=\beta$. Data covers the period of 1960:Q3 to 2018:Q4 and real GDP growth rate is com-

puted as $g_{t+h}=(400 / h) \operatorname{In}\left(y_{t+h} / y_{t}\right)$ where ${ }^{y_{t}}$ is the real GDP in levels forecasted h-quarters. The benchmark model is assumed to be $\mathrm{AR}(2)$ in equation 1.

On the forecast evaluation, we split and consider only $50 \%$ of the full data sample and subsequently obtain 1-, 2-, 4- and 8-quarters ahead out-of-sample forecasts based on the expanded window (recursive) approach. The predictive performance of the models is examined relative to the benchmark AR model using both point and density forecast evaluation tools. The former is the mean square forecast error (MSFE) [computed as the ayerage of the difference between forecast and real value of ${ }^{1}$ w while the latter is the average logarithm of predictive likelihoods (ALPL) computed as the logarithm of the predictive distribution evaluated over the out-of-sample period. The respective values of MSFE and ALPL are reported for the benchmark AR(2) model, whereas in the case of the alternative models, their values relative to that of the benchmark model are reported. Consequently, MSFE value that is less than one is considered to indicate

2 We thank Professor Gary Koop and Professor Dimitris Korobilis for sharing their Matlab codes and data used in this paper. support for the competing model over the benchmark, while value above one implies that the benchmark model performs better. Also, the smaller the value, the better the forecast of a specific model in comparison with other models. In the case of ALPL, values are expected to be positive to depict superior performance of the competing model than the benchmark, and larger for preference among the alternative models.

\section{RESULTS AND DISCUSSION}

The forecast performance of the various competing models is presented in Table 1. For convenience, the best model under each out-of-sample forecast horizon is in bold. We find that the models with the highest number of predictors (over 300 predictors) such as ELN/X and VBDVS/X account for most of the best forecast outcomes for real GDP growth albeit with distinct results for point and density forecasts. For instance, the ELN/X model offers the best point forecasts for two out of the four out-of-sample forecast periods while the VBDVS/X model does the same using density forecasts. Even for the remaining four out-of-sample forecast evaluations, models with about 60 predictors account for one each under point and density forecasts [i.e. SSVS/FAC60 for $\mathrm{h}=2$ under point forecast and VBDVS/FAC60 for $\mathrm{h}=4$ under density forecast] while the remaining two are shared between the benchmark model for the point forecast and a model with five predictors for the density forecast.

For robustness, we test further to see if the ability of the models with the large information sets to forecast US real GDP growth can be sustained using an alternative proxy namely the growth of industrial production index (IPI growth). The results of the latter are presented in Table 2 and the best forecast outcomes are captured in bold. Using the point forecast, the results are mixed as the forecast performance is shared equally between the five-factor (i.e. DMA/FAC5) model for $\mathrm{h}=1 \& \mathrm{~h}=2$ and the model with the largest information set (i.e. ELN/X) model for $\mathrm{h}=4 \mathrm{\&} \mathrm{h}=8$. However, for the density forecast, the output growth is better predicted with five-factor model involving the VBDVS/FAC5 which requires Dynamic Variable Selection prior with Variational Bayes. The outcome for the two proxies is not surprising though given what each is meant to measure. The real GDP growth is broader in scope than the IPI growth and therefore it is not unexpected to find more predictors required to produce accurate forecasts for the former than the latter. Finally, one consistent evidence from these analyses is that models that allow for Dynamic Variable Selection prior with Variational Bayes (VBDVS) as well as time-varying parameters tend to improve density forecasts.

\section{CONCLUSION}

This study considers an array of models and predictors to forecast US output growth over the period of 1960:Q1 to 2018:Q4. We employ both the Classical and Bayesian methods including the developed Dynamic Variable Selection prior with Variational Bayes [DVSVB] of Koop and Korobilis (2020) with over 300 predictors incrementally captured from 5 factors, 60 factors to over 300 factors. Overall, our results consistently support the use of high-dimensional models when forecasting US output growth regardless of the 
choice of forecast measure. The results however seem to favour the DVSVB with time varying parameter assumption when the density forecast of real GDP growth is evaluated.
An extension of this study that utilizes disaggregated (sectoral) output data will further enrich the literature on the subject.

Table 1. Point and Density Forecasts of Real GDP Growth.

\begin{tabular}{|c|c|c|c|c|c|c|c|c|}
\hline & \multicolumn{4}{|c|}{ MSFE } & \multicolumn{4}{|c|}{ ALPL } \\
\hline & $\mathrm{h}=1$ & $\mathrm{~h}=2$ & $\mathrm{~h}=4$ & $\mathrm{~h}=8$ & $\mathrm{~h}=1$ & $\mathrm{~h}=2$ & $\mathrm{~h}=4$ & $\mathrm{~h}=8$ \\
\hline \multicolumn{9}{|c|}{ MODEL WITH NO PREDICTORS } \\
\hline $\mathbf{A R}$ & 0.301 & 0.216 & 0.179 & 0.144 & 3.822 & 4.049 & 4.197 & 4.375 \\
\hline TVPAR & 1.180 & 1.503 & 1.629 & 1.489 & 0.361 & 0.322 & 0.255 & 0.179 \\
\hline \multicolumn{9}{|c|}{ MODEL WITH 5 PREDICTORS } \\
\hline FAC5 & 1.011 & 0.978 & 0.946 & 1.135 & 0.068 & 0.086 & 0.104 & -0.057 \\
\hline BAG/FAC5 & 0.979 & 0.957 & 0.987 & 1.178 & 0.104 & 0.118 & 0.117 & -0.013 \\
\hline GPR/FAC5 & 0.981 & 0.974 & 1.086 & 1.414 & 0.108 & 0.179 & 0.090 & -0.142 \\
\hline DMA/FAC5 & 0.927 & 0.878 & 0.911 & 1.070 & 0.225 & 0.192 & 0.148 & -0.024 \\
\hline VBDVS/FAC5 & 1.510 & 1.427 & 1.448 & 1.480 & 0.616 & 0.531 & -0.074 & -0.270 \\
\hline \multicolumn{9}{|c|}{ MODEL WITH 60 PREDICTORS } \\
\hline SSVS/FAC60 & 0.965 & 0.863 & 0.835 & 1.106 & 0.149 & 0.245 & 0.317 & 0.341 \\
\hline ELN/FAC60 & 0.929 & 0.944 & 0.870 & 1.039 & 0.149 & 0.268 & 0.358 & 0.425 \\
\hline VBDVS/FAC60 & 1.452 & 1.531 & 1.299 & 1.329 & 0.435 & 0.246 & 0.133 & 0.546 \\
\hline \multicolumn{9}{|c|}{ MODEL WITH 303 PREDICTORS } \\
\hline ELN/X & 0.910 & 0.962 & 0.772 & 1.214 & 0.203 & 0.322 & 0.280 & -0.020 \\
\hline PLS/X & 1.338 & 1.260 & 0.900 & 1.165 & -0.054 & 0.227 & 0.137 & 0.469 \\
\hline VBDVS/X & 1.079 & 1.092 & 1.122 & 1.077 & 0.478 & 0.597 & 0.593 & 0.445 \\
\hline
\end{tabular}

Note: MSFE is Mean Squared Forecast Error; ALPL is the Average Log-Predictive Likelihood. AR is the benchmark model involving two lags, estimated with OLS; TVPAR is a time-varying parameter version of the AR model, with stochastic volatility, estimated with MCMC; FAC5 builds on benchmark AR specification by augmenting it with first five principal components estimated with OLS; BAG/FAC5 involves the same predictors as FAC5, estimated as constant parameter regression using Bagging; DMA/FAC5 involves same predictors as FAC5, estimated as TVP regression using Dynamic Model Averaging; VBDVS/FAC5, same predictors as FAC5, estimated as TVP regression using our Dynamic Variable Selection prior with Variational Bayes; GPR/FAC5, same predictors as FAC5, estimated as a Gaussian Process Regression; SSVS/FAC60, builds on benchmark AR specification by augmenting it with first 60 principal components, estimated using an SSVS prior with MCMC; ELN/FAC60, same predictors as SSVS/FAC60, estimated as a constant parameter regression using the Elastic Net; VBDVS/FAC60, same predictors as SSVS/FAC60, estimated as a TVP regression using Koop and Korobilis (2020) Dynamic Variable Selection prior with Variational Bayes; ELN/X builds on benchmark AR specification by augmenting it with 303 predictors, estimated using the Elastic Net; PLS/X has the same predictors as in ELN/X, estimated as a constant parameter Partial Least Squares regression; VBDVS/X has the same predictors as in ELN/X, estimated as a TVP regression using Koop and Korobilis (2020) Dynamic Variable Selection prior with Variational Bayes.

Table 2. Point and Density Forecasts of IPI Growth.

\begin{tabular}{|c|c|c|c|c|c|c|c|c|}
\hline & \multicolumn{4}{|c|}{ MSFE } & \multicolumn{4}{|c|}{ ALPL } \\
\hline & $\mathrm{h}=1$ & $\mathrm{~h}=2$ & $\mathrm{~h}=4$ & $\mathrm{~h}=8$ & $\mathrm{~h}=1$ & $\mathrm{~h}=2$ & $\mathrm{~h}=4$ & $\mathrm{~h}=8$ \\
\hline \multicolumn{9}{|c|}{ MODEL WITH NO PREDICTORS } \\
\hline $\mathbf{A R}$ & 0.768 & 0.742 & 0.858 & 0.643 & 3.409 & 3.505 & 3.574 & 3.746 \\
\hline TVPAR & 1.173 & 1.537 & 2.420 & 2.210 & 0.379 & 0.223 & 0.517 & 0.891 \\
\hline \multicolumn{9}{|c|}{ MODEL WITH 5 PREDICTORS } \\
\hline FAC5 & 0.871 & 0.890 & 0.951 & 1.149 & 0.061 & 0.089 & 0.129 & 0.247 \\
\hline BAG/FAC5 & 0.941 & 0.959 & 1.046 & 1.157 & 0.056 & 0.083 & 0.116 & 0.223 \\
\hline GPR/FAC5 & 0.879 & 0.944 & 1.011 & 1.275 & 0.086 & 0.112 & 0.175 & 0.280 \\
\hline
\end{tabular}




\begin{tabular}{|c|c|c|c|c|c|c|c|c|c|c|}
\hline DMA/FAC5 & 0.824 & 0.884 & 0.916 & 1.132 & 0.247 & 0.150 & 0.100 & 0.114 \\
\hline VBDVS/FAC5 & 1.037 & 1.722 & 2.724 & 1.517 & 0.503 & 0.592 & 0.511 & 0.562 \\
\hline \multicolumn{7}{|c|}{ MODEL WITH 60 PREDICTORS } \\
\hline SSVS/FAC60 & 1.002 & 1.023 & 0.996 & 0.941 & 0.081 & 0.128 & 0.212 & 0.299 \\
\hline ELN/FAC60 & 0.974 & 1.186 & 1.128 & 1.005 & 0.096 & 0.148 & 0.166 & 0.337 \\
\hline VBDVS/FAC60 & 0.932 & 1.272 & 1.718 & 1.592 & 0.536 & 0.314 & 0.079 & -0.133 \\
\hline \multicolumn{7}{|c|}{} & & MODEL WITH 303 PREDICTORS & \multicolumn{3}{|c|}{0} \\
\hline ELN/X & 1.040 & 0.989 & 0.742 & 0.898 & 0.249 & 0.317 & 0.421 & -0.259 \\
\hline PLS/X & 1.353 & 1.285 & 1.049 & 1.055 & -0.013 & 0.166 & -0.035 & 0.329 \\
\hline VBDVS/X & 1.061 & 1.058 & 1.544 & 1.296 & 0.464 & 0.477 & 0.653 & -0.180 \\
\hline
\end{tabular}

Note: MSFE is Mean Squared Forecast Error; ALPL is the Average Log-Predictive Likelihood. AR is the benchmark model involving two lags, estimated with OLS; TVPAR is a time-varying parameter version of the AR model, with stochastic volatility, estimated with MCMC; FAC5 builds on benchmark AR specification by augmenting it with first five principal components estimated with OLS; BAG/FAC5 involves the same predictors as FAC5, estimated as constant parameter regression using Bagging; DMA/FAC5 involves same predictors as FAC5, estimated as TVP regression using Dynamic Model Averaging; VBDVS/FAC5, same predictors as FAC5, estimated as TVP regression using our Dynamic Variable Selection prior with Variational Bayes; GPR/FAC5, same predictors as FAC5, estimated as a Gaussian Process Regression; SSVS/FAC60, builds on benchmark AR specification by augmenting it with first 60 principal components, estimated using an SSVS prior with MCMC; ELN/FAC60, same predictors as SSVS/FAC60, estimated as a constant parameter regression using the Elastic Net; VBDVS/FAC60, same predictors as SSVS/FAC60, estimated as a TVP regression using Koop and Korobilis (2020) Dynamic Variable Selection prior with Variational Bayes; ELN/X builds on benchmark AR specification by augmenting it with 303 predictors, estimated using the Elastic Net; PLS/X has the same predictors as in ELN/X, estimated as a constant parameter Partial Least Squares regression; VBDVS/X has the same predictors as in ELN/X, estimated as a TVP regression using Koop and Korobilis (2020) Dynamic Variable Selection prior with Variational Bayes.

\section{CONFLICT OF INTEREST STATEMENT}

The authors declare that they have no conflict of interest.

\section{REFERENCES}

Aromi, D.J. (2019). Medium term growth forecasts: Experts vs. simple models. International Journal of Forecasting, 35, 1085-1099.

Baghestani, H. \& AbuAl-Fou, B.M. (2017). Comparing Federal Reserve, Blue Chip, and time series forecasts of US output growth. Journal of Economics and Business, 89, 47-56.

Breiman, L. (1996). Bagging Predictors. Machine Learning, 24, 123-140.

Ercolani, V. (2020). Forecasting US recessions: The role of economic uncertainty. Economics Letters, 193, 109302.
George, E. I. and R. E. McCulloch (1993). Variable Selection via Gibbs Sampling. Journal of the American Statistical Association, 88, 881889.

Koop, G. and D. Korobilis (2012). Forecasting Inflation Using Dynamic Model Averaging. International Economic Review, 53, 867-886.

Koop, G., and Korobilis, D. (2020). Bayesian dynamic variable selection in high dimensions. Available at SSRN: https://ssrn.com/abstract=3246472.

Zou, H. and T. Hastie (2005). Regularization and Variable Selection via the Elastic Net. Journal of the Royal Statistical Society. Series B (Statistical Methodology), 67, 301-320. 